This is an electronic reprint of the original article. This reprint may differ from the original in pagination and typographic detail.

\author{
Author(s): Chen, Yan-Cong; Liu, Jun-Liang; Lan, Yanhua; Zhong, Zhi-Qiang; Mansikkamäki, Akseli; \\ Ungur, Liviu; Li, Quan-Wen; Jia, Jian-Hua; Chibotaru, Liviu F.; Han, Jun-Bo; \\ Wernsdorfer, Wolfgang; Chen, Xiao-Ming; Tong, Ming-Liang
}

Title: $\quad$ Dynamic Magnetic and Optical Insight into a High Performance Pentagonal Bipyramidal Dy(III) Single-lon Magnet

Year: $\quad 2017$

Version:

Please cite the original version:

Chen, Y.-C., Liu, J.-L., Lan, Y., Zhong, Z.-Q., Mansikkamäki, A., Ungur, L., Li, Q.-W., Jia, J.-H., Chibotaru, L. F., Han, J.-B., Wernsdorfer, W., Chen, X.-M., \& Tong, M.-L. (2017). Dynamic Magnetic and Optical Insight into a High Performance Pentagonal Bipyramidal Dy(III) Single-Ion Magnet. Chemistry: A European Journal, 23(24), 57085715. https://doi.org/10.1002/chem.201606029

All material supplied via JYX is protected by copyright and other intellectual property rights, and duplication or sale of all or part of any of the repository collections is not permitted, except that material may be duplicated by you for your research use or educational purposes in electronic or print form. You must obtain permission for any other use. Electronic or print copies may not be offered, whether for sale or otherwise to anyone who is not an authorised user. 


\section{CHEMISTRY A European Journal}

\section{Accepted Article}

Title: Dynamic Magnetic and Optical Insight into a High Performance Pentagonal Bipyramidal Dy(III) Single-lon Magnet

Authors: Yan-Cong Chen, Jun-Liang Liu, Yanhua Lan, Zhi-Qiang Zhong, Akseli Mansikkamäki, Liviu Ungur, Quan-Wen Li, Jian-Hua Jia, Liviu F. Chibotaru, Jun-Bo Han, Wolfgang Wernsdorfer, Xiao-Ming Chen, and Ming-Liang Tong

This manuscript has been accepted after peer review and appears as an Accepted Article online prior to editing, proofing, and formal publication of the final Version of Record (VoR). This work is currently citable by using the Digital Object Identifier (DOI) given below. The VoR will be published online in Early View as soon as possible and may be different to this Accepted Article as a result of editing. Readers should obtain the VoR from the journal website shown below when it is published to ensure accuracy of information. The authors are responsible for the content of this Accepted Article.

To be cited as: Chem. Eur. J. 10.1002/chem.201606029

Link to VoR: http://dx.doi.org/10.1002/chem.201606029 


\title{
Dynamic Magnetic and Optical Insight into a High Performance Pentagonal Bipyramidal Dy(III) Single-Ion Magnet
}

\author{
Yan-Cong Chen, ${ }^{[a]}$ Jun-Liang Liu, ${ }^{*[a]}$ Yanhua Lan, ${ }^{[b]}$ Zhi-Qiang Zhong, ${ }^{\left[{ }^{[c]}\right.}$ Akseli Mansikkamäki, ${ }^{[d, e]}$ Liviu \\ Ungur, ${ }^{[d, f]}$ Quan-Wen Li, ${ }^{[a]}$ Jian-Hua Jia, ${ }^{[a]}$ Liviu F. Chibotaru, ${ }^{[d]}$ Jun-Bo Han, ${ }^{[c]}$ Wolfgang Wernsdorfer, ${ }^{[b]}$ \\ Xiao-Ming Chen, ${ }^{[\mathrm{a}]}$ and Ming-Liang Tong ${ }^{*[\mathrm{a}]}$
}

\begin{abstract}
The pentagonal bipyramidal single-ion magnets (SIMs) are among the most attractive prototypes of high performance single-molecule magnets (SMMs). Here we introduced the fluorescence-active phosphine oxide ligand, combing the dynamic magnetic measurement, optical characterization and ab initio calculation, firstly studied the magneto-optical correlation of a highperformance pseudo- $D_{5 h}$ Dy(III) SIM with large $U_{\text {eff }}=508(2) \mathrm{K}$ and high magnetic hysteresis temperature of $19 \mathrm{~K}$, $\left[\mathrm{Dy}\left(\mathrm{CyPh}_{2} \mathrm{PO}\right)_{2}\left(\mathrm{H}_{2} \mathrm{O}\right)_{5}\right] \mathrm{Br}_{3} \cdot 2\left(\mathrm{CyPh}{ }_{2} \mathrm{PO}\right) \cdot \mathrm{EtOH} \cdot 3 \mathrm{H}_{2} \mathrm{O} \quad\left(\mathrm{CyPh}_{2} \mathrm{PO}=\right.$ cyclohexyl(diphenyl)phosphine oxide). This work shall provide a deeper insight into the rational design of promising molecular magnets.
\end{abstract}

\section{Introduction}

Single-molecule magnets have attracted strong interest in recent years, as it can be trapped in one of the bistable magnetic states that separated by an energy barrier. Among the most promising candidates for high-density information storage, quantum processing and spintronics, ${ }^{[1]}$ a good SMM shall combine the large energy barrier, high blocking temperature and weak quantum tunneling of magnetization (QTM). The early cases of polymetallic clusters of transition metals show highly suppressed QTM, ${ }^{[2]}$ but the increasing of the energy barrier hits the bottleneck as the difficulty of combining the large spin states and

[a] Y.-C. Chen, Dr. J.-L., Liu, Q.-W. Li, Dr. J.-H. Jia, Prof. Dr. X.-M. Chen, Prof. Dr. M.-L. Tong

Key Laboratory of Bioinorganic and Synthetic Chemistry of Ministry of Education, School of Chemistry, Sun Yat-Sen University, Guangzhou 510275, P. R. China

E-mail: tongml@mail.sysu.edu.cn

[b] Dr. Y. Lan, Prof. Dr. W. Wernsdorfer

Institut Néel, CNRS \& Université Joseph Fournier, BP 166, 25 avenue des Martyrs, 38042 Grenoble Cedex 9, France

[c] Z.-Q. Zhong, Prof. Dr. J.-B. Han

Wuhan National High Magnetic Center, Huazhong University of Science and Technology, Wuhan 430074, P. R. China

[d] A. Mansikkamäki, Dr. L. Ungur, Prof. Dr. L. F.Chibotaru Theory of Nanomaterials Group and INPAC-Institute of Nanoscale Physics and Chemistry, Katholieke, Universiteit Leuven, Celestijnenlaan 200F, 3001 Leuven, Belgium

[e] A. Mansikkamäki

Department of Chemistry, Nanoscience Center, University of Jyväskylä, P. O. Box 35, 40014 Jyväskylä, Finland

[f] Dr. L. Ungur

Theoretical Chemistry, Lund University, Getingevagen 60, 22201 Lund, Sweden.

Supporting information for this article is given via a link at the end of the document. high anisotropies together. Lanthanide metal ions, especially $\mathrm{Tb}(\mathrm{III}), \mathrm{Dy}(\mathrm{III}), \mathrm{Ho}(\mathrm{III}), \mathrm{Er}(\mathrm{III})^{[3]}$ with intrinsic large spin-orbit interactions, have been extensively studied recently for SMMs, and the radical-bridged dilanthanide complexes achieved a high blocking temperature up to $14 \mathrm{~K} \cdot{ }^{[4]}$.

For most of the high performance monometallic lanthanide SMMs, a smart strategy is to stabilize some typical coordination symmetries for reducing the off-diagonal elements of orbit-lattice interaction matrix and therefore, suppressing QTM. [3b, 3e, 5] Based on crystal-field theory, ${ }^{[6]}$ besides square antiprismatic complexes toward $D_{4 \mathrm{~d}}$ symmetry $\left[{ }^{[b}, 5 \mathrm{e}-\mathrm{f}, \quad 7\right]$ and linear 2coordinated complexes ${ }^{[5 a-c]} /$ sandwich-type complexes toward $D_{\infty \mathrm{h}}$ symmetry, ${ }^{[3 \mathrm{~d}, 8]}$ pentagonal bipyramidal complexes are on the way of getting close to $D_{5 \mathrm{~h}}$ symmetry that suppresses quantum tunneling and exhibits record anisotropic barriers. These facts illustrate that pseudo- $D_{5 h}$-symmetry SIMs are highly attractive, and it is worth deeply understanding the magnetic dynamics and energy structures. ${ }^{[9]}$ Although the strict 5 -fold symmetry is forbidden in conventional crystals, researchers have been trying to push the local symmetry to the limit. ${ }^{[9-10]}$

Recently, we investigated the pentagonal bipyramidal Dy(III) SIMs which comprise a highly symmetrical $\left[\mathrm{Dy}\left(\mathrm{Cy}_{3} \mathrm{PO}\right)_{2}\left(\mathrm{H}_{2} \mathrm{O}\right)_{5}\right]^{3+}$ $\left(\mathrm{Cy}_{3} \mathrm{PO}=\right.$ tricyclohexyl phosphine oxide $)$ core. ${ }^{[9 \mathrm{c}]}$ The strong axial crystal field provided by the phosphine oxide, combined with the weak equatorial ligands arranged in pseudo-5-fold symmetry, lead to the energy barriers around $500 \mathrm{~K}$ and a record-breaking magnetic hysteresis temperature up to $20 \mathrm{~K}$ for the $\mathrm{Br}$ derivative. To the best of our knowledge, it is also the first report on the Ln-SIMs based on phosphine oxide, which is undoubtedly an emerging and promising family in this area and requires deeper understanding on their multifaceted behaviors and magneto-structural correlations.

Based on the previous study, we introduced the aromatic substituent groups into the system as sensitive antennas to promote the luminescence of Dy(III) ions. Indeed, the fluorescence spectra of rare earth ions can provide extremely valuable information on the energy levels, which is a direct probe and also a solid support to the magnetic studies and the $a b$ initio calculations. The title complex here, $\left[\mathrm{Dy}\left(\mathrm{CyPh}_{2} \mathrm{PO}\right)_{2}\left(\mathrm{H}_{2} \mathrm{O}\right)_{5}\right] \mathrm{Br}_{3} \cdot 2\left(\mathrm{CyPh}_{2} \mathrm{PO}\right) \cdot \mathrm{EtOH} \cdot 3 \mathrm{H}_{2} \mathrm{O}\left(\mathbf{1}, \mathrm{CyPh}_{2} \mathrm{PO}\right.$ $=$ cyclohexyl(diphenyl)phosphine oxide), comprise a $\left[\mathrm{Dy}\left(\mathrm{R}_{3} \mathrm{PO}\right)_{2}\left(\mathrm{H}_{2} \mathrm{O}\right)_{5}\right]^{3+}$ analogue core that retains the nice SMM properties. In addition, the advantages of $\mathbf{1}$ compare with its predecessor includes: 1) A further increased symmetry by a $\mathrm{C}_{2}$ axis through the Dy(III) ion; 2) The feasibility to obtain suitable single crystals for micro-SQUID measurement; 3) The introduction of aromatic substituent groups to yield prominent fluorescence spectra for luminescence study. By a 
comprehensive study of crystallography, dc/ac magnetic characterization, single crystal micro-SQUID measurement and luminescence study, we managed to have a deeper understanding of the magneto-structural correlation, the relaxation dynamics and the QTM mechanism of SMMs. These combined with the state-of-the-art ab initio calculation shall shed light onto the further study on SMMs.

\section{Results and Discussion}

\section{Crystal Structure}

The title compound can be synthesized from $\mathrm{DyBr}_{3}$ and $\mathrm{CyPh}_{2} \mathrm{PO}$ in the $\mathrm{H}_{2} \mathrm{O} / \mathrm{EtOH}$ mixed solution followed by recrystallization in $\mathrm{EtOH}$ and slow evaporation in ambient condition (see experimental section for details). Single-crystal crystallography shows that $\mathbf{1}$ crystallizes in the orthorhombic space group $C 222_{1}$ and each asymmetric unit contains one $\mathrm{Dy}^{3+}$ ion, two coordinated $\mathrm{CyPh}_{2} \mathrm{PO}$ ligands in the opposite direction, five coordinated waters in the equatorial plane, two free ligand, three $\mathrm{Br}^{-}$ions in the outer coordination spheres and two $\mathrm{EtOH}$ molecules (Fig. 1). An additional symmetry of a $\mathrm{C}_{2}$ axis (compared with the $\mathrm{Cy}_{3} \mathrm{PO}$ analogue) through the Dy1-O1W provides the crystallographic guarantee for the equal bond lengths and angles for the main anisotropy axis along O1-DyO1A, which can be regarded as a step further toward the ideal pentagonal bipyramid geometry. Indeed, the value of Continuous Shape Measures calculations (CShM) is 0.174 , which is only slightly larger than the most perfect pseudo- $D_{5 \mathrm{~h}}$ symmetry Ln-SIM (CShM = 0.142, [Dy$\left.\left.\left(\mathrm{Cy}_{3} \mathrm{PO}\right)_{2}\left(\mathrm{H}_{2} \mathrm{O}\right)_{5}\right] \mathrm{Br}_{3} \cdot 2\left(\mathrm{Cy}_{3} \mathrm{PO}\right) \cdot 2 \mathrm{H}_{2} \mathrm{O} \cdot 2 \mathrm{EtOH}\right)^{[9 c]}$ reported to date (Table S2). Nevertheless, the actual symmetry for $\mathbf{1}$ is only $\mathrm{C}_{2}$.
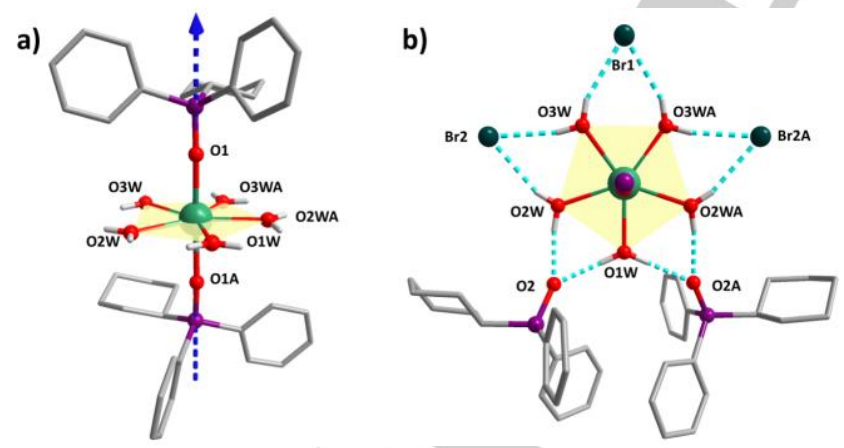

Figure 1. Crystal Structures of 1 emphasizing the pseudo-five-fold symmetry. a): Coordination environment of Dy(III) in 1. The blue dash line represents the orientation of the main magnetic axes of the lowest Kramers doublets. b): Outer coordination sphere connected with hydrogen bonds in $1 . \mathrm{H}$ atoms of $\mathrm{CyPh}_{2} \mathrm{PO}$ are omitted for clarity. Color Codes: Dy, green; $\mathrm{P}$, purple; $\mathrm{Br}$, cyan; $\mathrm{O}$, red; C, gray; H, light gray. Symmetry code (A): 1-x, y, 0.5-z.

For the outer coordination sphere, a five-pointed star is formed by hydrogen bonds between the five coordinated $\mathrm{H}_{2} \mathrm{O}$ molecules, three $\mathrm{Br}$ ions and two free ligands (Fig. 1b), which helps the stabilization of the pseudo- $D_{5 \mathrm{~h}}$ symmetry of the first coordination sphere. Overall the local environment of $\mathrm{Dy}^{3+}$ in $\mathbf{1}$ is a highly compressed pentagonal bipyramid with the average DyO distances of $2.217 \AA$ (axial) and $2.364 \AA$ (equatorial). Face-toface and edge-to-face $\pi-\pi$ stacking are formed between the benzyl rings of the coordinated ligands and the uncoordinated ones. Between the discrete molecules, the nearest Dy-Dy distance in the crystal structure is as far as $13.8 \AA$ and there is no direct or super-exchange interactions through chemical bonds.

\section{Magnetic Characterization}

Variable-temperature magnetic susceptibilities were measured on polycrystalline samples of 1 under a $0.1 \mathrm{~T}$ dc field (Fig. 2a). At room temperature, the $\chi_{M} T$ value is $13.9 \mathrm{~cm}^{3} \mathrm{~K} \mathrm{~mol}^{-1}$, a little lower than the expected value for a free $D y^{3+}$ ion $\left(14.17 \mathrm{~cm}^{3} \mathrm{~K}\right.$ $\mathrm{mol}^{-1}$ ). Upon cooling, $\chi_{\mathrm{M}} T$ gradually decrease and then suddenly drop below ca. $12 \mathrm{~K}$. Meanwhile, the zero-field-cooled / fieldcooled (ZFC-FC) magnetization under a $0.1 \mathrm{~T}$ in warm mode $(2$ $\mathrm{K} / \mathrm{min}$ ) (inset of Fig. 2a) shows a clear divergence below $11 \mathrm{~K}$ with a peak at $10.5 \mathrm{~K}$, indicating the process into magnetic blocking. The approaching magnetization values to $5 N \beta$ and the overlapped $M$ vs. $H / T$ curves (Fig. S5) provide an evidence of very far-separated excited doublets for $\mathrm{Dy}^{3+}$.
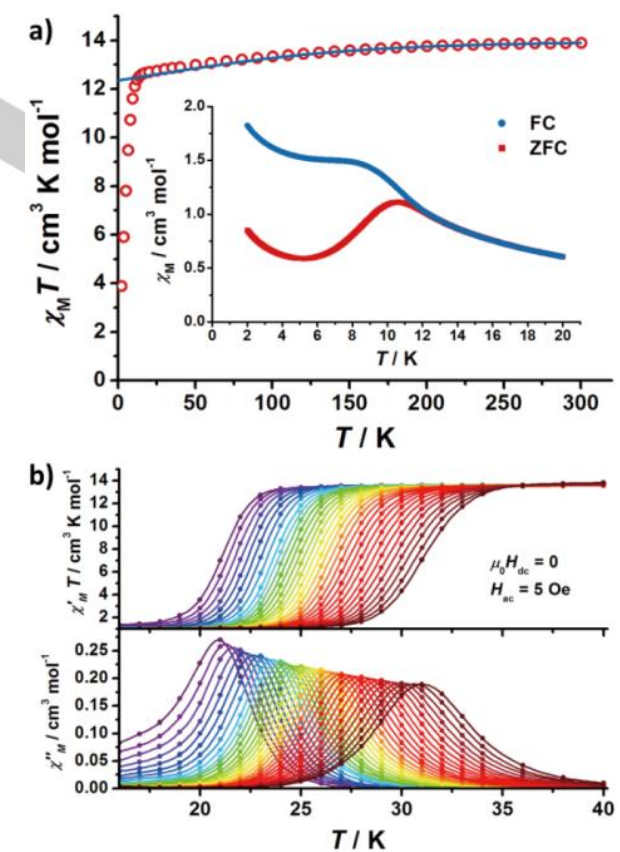

Figure 2. a) Temperature dependence of the molar magnetic susceptibility $X M T$ products and the zero-field-cooled / field-cooled (ZFC-FC) magnetic susceptibilities (inset) for 1 under a $0.1 \mathrm{~T}$ dc field sweeping at $2 \mathrm{~K} / \mathrm{min}$ in warming mode. The solid line corresponds to the ab initio calculations. b) Temperature dependence of the in-phase $X^{\prime} M T$ product and out-of-phase $X{ }^{\prime \prime} M$ for 1 in zero dc field with the ac frequency of $1-1488 \mathrm{~Hz}$.

To reveal the dynamic magnetic behaviors, variabletemperature and variable-frequency ac magnetic susceptibilities were measured in zero dc field and a $0.1 \mathrm{~T}$ dc field. From the temperature-dependent data (Fig. 2b), the patterns are quite 
typical for SMMs, with the $1488 \mathrm{~Hz}$ peak located at $31 \mathrm{~K}$ down to the $1 \mathrm{~Hz}$ peak located at $21 \mathrm{~K}$. No overwhelming QTM can be observed on the ac data in the measured window.

For the frequency-dependent measurement (Fig. 3), we pushed the low-frequency limit to $0.1 \mathrm{~Hz}$ and the ac peaks still keep shifting towards low frequency region. Moreover, the ac susceptibilities are almost unaffected by the application of a 0.1 $\mathrm{T}$ dc field (Fig. 3b). For the high temperature region, the temperature dependence of the relaxation time $\tau$ perfectly obeys the Arrhenius law corresponding to the Orbach process, and the best fit gives the $U_{\text {eff }}=508(2) \mathrm{K}, T_{0}=8.6 \times 10^{-12} \mathrm{~s}$ for zero dc field and $U_{\text {eff }}=503(4) \mathrm{K}, T_{0}=9.5 \times 10^{-12} \mathrm{~s}$ for $0.1 \mathrm{~T}$ dc field (Fig. 4a).
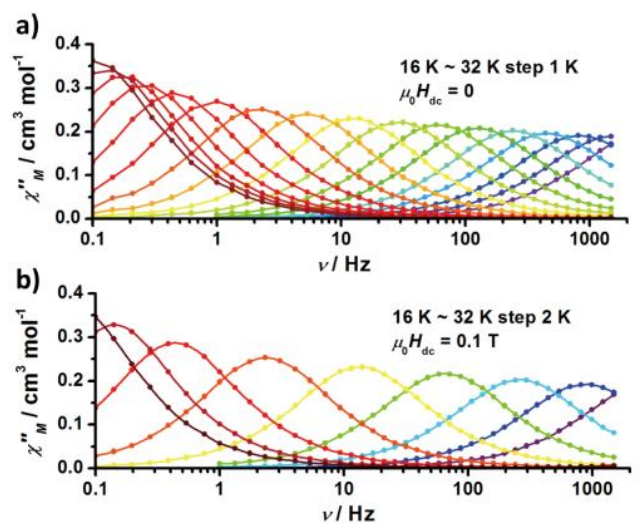

Figure 3. Frequency dependence of the out-of-phase $X{ }^{\prime \prime} M$ in zero dc field (a) and $0.1 \mathrm{~T}$ dc field $(\mathrm{b})$ for 1 . Lines are guides to the eyes.

In order to investigate the slow relaxation behavior at lower temperatures, we managed to extend the measured relaxation time using dc magnetization decay. The relaxation dynamics can be fitted with the multiple relaxation equation $T^{-1}=T_{0}{ }^{-1} \exp \left(-U_{\text {eff }}\right.$ $\left./ k_{B} T\right)+C T^{n}+T_{\mathrm{QTM}}{ }^{-1}$ involving a Raman process with $n=4.0(2)$ and slowly goes to a platform with $T_{Q T M}=19.5(8) \mathrm{s}$ for zero dc field. When the final dc field is set as $0.1 \mathrm{~T}$, the relaxation times keeps rising with $n=5.61(4)$ and goes up to $1775 \mathrm{~s}$ at $5 \mathrm{~K}$, showing the suppression of QTM (Fig. 4a).

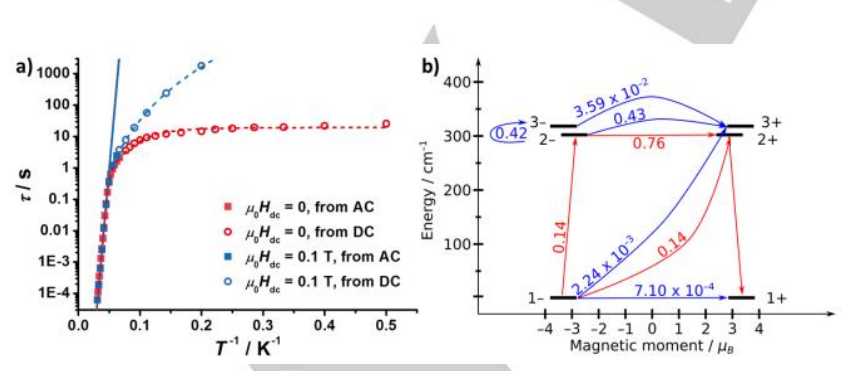

Figure 4. a) Temperature dependence of the relaxation time $r$ in zero dc field (red) and a $0.1 \mathrm{~T}$ Oe dc field (blue) for 1 . The solid lines are the best fits to the Arrhenius law, while the dashed lines are the best fit to the multiple relaxation equation. b) Magnetization blocking barrier of $\mathbf{1}$. The lowest spin-orbit states are arranged according to the magnitude of their magnetic moments on the horizontal axis. The numbers next to arrows connecting two states display the average transition magnetic moment matrix element between the respective states. The most probable relaxation route is indicated by red arrows and takes place via the first excited Kramers doublet.

Magnetic hysteresis loops can be observed as the extremely slow magnetic relaxation rate at low temperatures, even on a conventional PPMS-VSM (Fig. 5). The butterfly shape is typical, due to the faster relaxation around zero field and the major reasons are the unsuppressed QTM from: 1) the deviation of the symmetry; 2) the hyperfine interaction and 3) the dipolar interactions. The hysteresis loops become narrower with the increase of temperature, and the remanent magnetization $\left(M_{r}\right)$ and coercive field $\left(H_{c}\right)$ also become smaller (Fig. S7). Conservatively, we consider the hysteresis temperature to be 19 $\mathrm{K}$ with the field ramping speed of $0.02 \mathrm{~T} / \mathrm{s}$ (In higher temperatures the measurement falls in instrumental error when $H_{c}<0.01 \mathrm{~T}$.), which is comparable with the $\mathrm{Cy}_{3} \mathrm{PO}$ derivative ${ }^{[9 c]}$ and higher than that of [Dy(bbpen) $\mathrm{Br}]{ }^{[9 \mathrm{dd}]}$ It should be noted that the hysteresis temperature is usually different from the peak temperature of ZFC susceptibilities. Indeed, there will not be a strict definition of blocking temperatures for SMMs but depends on different measurements, as the slow relaxation of magnetization is not a real "blocking" like those in the phase changing materials. Here we also notice that some of the extremely high energy barriers do not guarantee the proportional increase of the relaxation times, ${ }^{[9 \mathrm{~d}, 10 \mathrm{~b}-\mathrm{c}]}$ as the relaxation is already dominated by Raman process in lower temperature region.
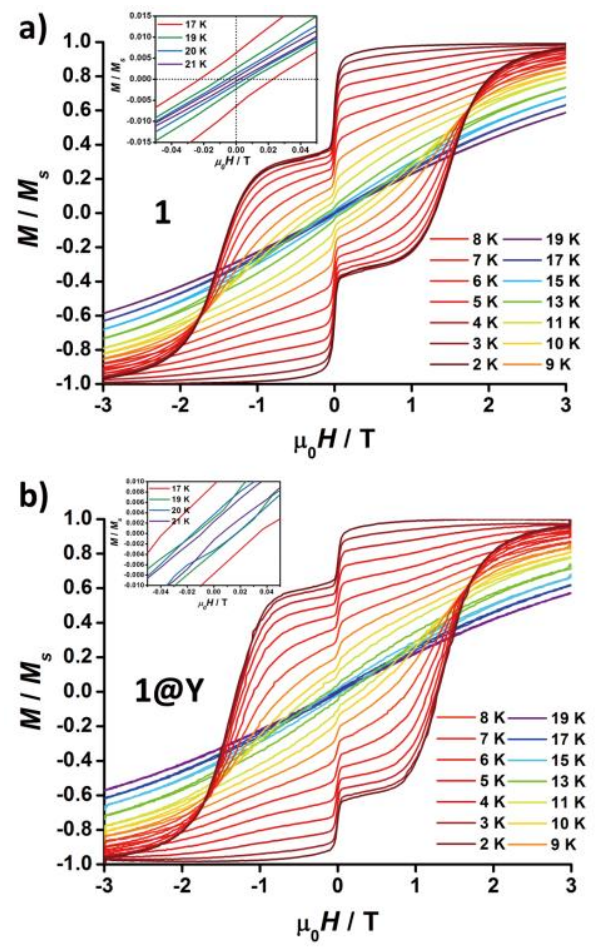

Figure 5. Normalized magnetic hysteresis loops for 1 (a) and $1 @ Y$ (b). The data were continuously collected at intervals of $1 \mathrm{~s}$ with the field ramping speed of $0.02 \mathrm{~T} / \mathrm{s}$ at various temperatures. 
Further study of the diluted sample 1@Y does not show much difference from 1. Fitting the ac susceptibilities yield the consistent $U_{\text {eff }}=467(5) \mathrm{K}, T_{0}=2.4 \times 10^{-11} \mathrm{~s}$ for zero dc field and $U_{\text {eff }}=465(5) \mathrm{K}, t_{0}=2.4 \times 10^{-11} \mathrm{~s}$ for a $0.1 \mathrm{~T}$ dc field (Fig. S9-11) . The divergence of ZFC-FC curves is also observed below $11 \mathrm{~K}$ with a peak at $10.5 \mathrm{~K}$ (Fig. S12). The remanent magnetization $\left(M_{r}\right)$ becomes larger after the dilution (Fig. 5b), which be attributed to the weakening of intermetallic magnetic interactions, though the magnetic hysteresis loops still keep open up to $19 \mathrm{~K}$.

\section{Micro-SQUID Measurement}

To extend the characterization down to ultra-low temperature, micro-SQUID measurements were performed on a single-crystal of 1. The hysteresis loops at $4 \mathrm{~K}$ show the similar shapes as the powder sample (Fig. 6a), which become even more prominent at $0.03 \mathrm{~K}$ (Fig. 6b). For the decay of the DC magnetization at zero applied field, the single-crystal measurements should be comparable to the powder measurements and the relaxation time obtained from these two measurements should be converged in the same temperature regime. As illustrated in the plots (Fig. 6d), although the two datasets follow in the same trend, they could be converged only with a multiplying factor of 1.7. Such a difference could be due to the fact that there is no way to set the field exactly as zero in conventional SQUID and the remanent field always exists.
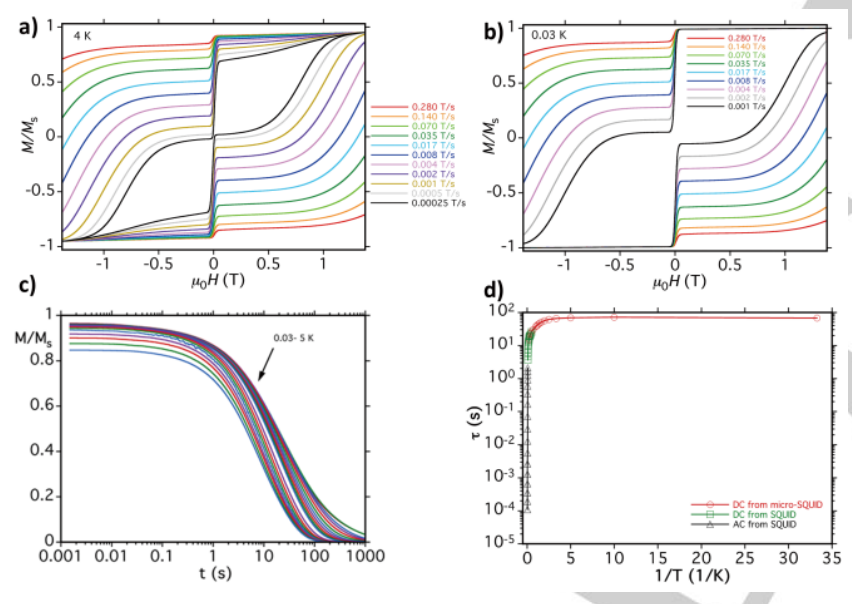

Figure 6. Dynamic magnetic properties for for 1 measured on micro-SQUID: a) Normalized magnetic hysteresis loops at $4 \mathrm{~K}$; b) Normalized magnetic hysteresis loops at $0.03 \mathrm{~K}$; c) The decay of the normalized DC magnetization at various temperatures.d) Temperature dependence of the relaxation time $I$ compared with the AC (black) and DC (green) data from SQUID.

\section{Luminescene Study}

The introduction of aromatic substituent groups in $\mathbf{1}$ leads to nice fluorescence spectra both at room temperature and at low temperature, which provides much more valuable information on the energy levels. In general, the emission spectrum of 1 shows the characteristic peaks for Dy(III) with three groups of sharp peaks corresponding to the $\mathrm{f}-\mathrm{f}$ transition around $475 \mathrm{~nm}(21000$ $\left.\mathrm{cm}^{-1},{ }^{4} \mathrm{~F}_{9 / 2} \rightarrow{ }^{6} \mathrm{H}_{15 / 2}\right), 575 \mathrm{~nm}\left(17400 \mathrm{~cm}^{-1},{ }^{4} \mathrm{~F}_{9 / 2} \rightarrow{ }^{6} \mathrm{H}_{13 / 2}\right)$ and 670 $\mathrm{nm}\left(15000 \mathrm{~cm}^{-1}{ }^{4} \mathrm{~F}_{9 / 2} \rightarrow{ }^{6} \mathrm{H}_{11 / 2}\right)$. (Fig. S13). Here we focus on the
${ }^{4} \mathrm{~F}_{9 / 2} \leftrightarrow{ }^{6} \mathrm{H}_{15 / 2}$ transitions around $21000 \mathrm{~cm}^{-1}$ involving the lowestlying energy levels that are crucial to the magnetic dynamics (Fig. 7).

At room temperature, it seems difficult to recognize the multiple peaks. Then, on lowering the temperature, the ${ }^{6} \mathrm{H}_{15 / 2} \rightarrow{ }^{4} \mathrm{~F}_{9 / 2}$ absorption and the ${ }^{6} \mathrm{H}_{15 / 2} \rightarrow{ }^{4} \mathrm{~F}_{9 / 2} \rightarrow{ }^{6} \mathrm{H}_{13 / 2}$ excitation spectra become sharper, and we noticed that the peaks at $20983 \mathrm{~cm}^{-1}$ (No. 2) and $20914 \mathrm{~cm}^{-1}$ (No. 3) disappear, indicating the depopulation of excited states. At $10 \mathrm{~K}$, the last peak of the absorption and excitation spectra overlap perfectly with the first peak of the emission spectra at $21308 \mathrm{~cm}^{-1}$ (No. 1), which is assigned as the ground-to-ground transition of ${ }^{4} \mathrm{~F}_{9 / 2} \leftrightarrow{ }^{6} \mathrm{H}_{15 / 2}$ (red arrow in Fig. 7b). Then, the eight Kramers doublets from the splitting of ${ }^{6} \mathrm{H}_{15 / 2}$ multiplet can be successively determined from the fine structure of the ${ }^{4} \mathrm{~F}_{9 / 2} \rightarrow{ }^{6} \mathrm{H}_{15 / 2}$ emission. Meanwhile, we believe the huge intensity difference of the emission peaks also correspond to the extent of mixing of the wave functions, as these transitions are intrinsically forbidden while the selection rules can be broken by lanthanide-crystal field interactions.

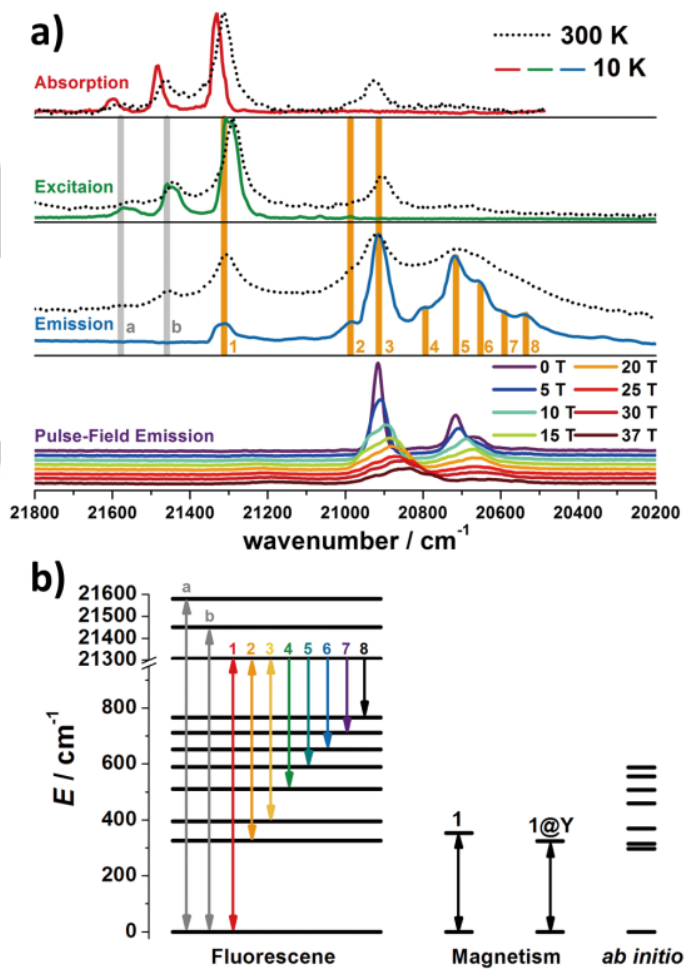

Figure 7. a) Fluorescence Spectra of 1 showing the ${ }^{4} \mathrm{~F}_{9 / 2} \leftrightarrow{ }^{6} \mathrm{H}_{15 / 2}$ peaks. The absorption, excitation $\left(\lambda_{\mathrm{em}}=574 \mathrm{~nm}\right)$ and emission $\left(\lambda_{\mathrm{ex}}=361 \mathrm{~nm}\right)$ spectra were measured at $300 \mathrm{~K}$ (black dotted lines) and $10 \mathrm{~K}$ (color solid lines), and the pulse-field emission were measured at $5 \mathrm{~K}$. Vertical lines indicate the optical transitions between the microstates of ${ }^{4} \mathrm{~F}_{9 / 2}$ and ${ }^{6} \mathrm{H}_{15 / 2}$ and the emission spectra are plotted in logarithmic intensity for clarity. b) Energy levels determined by the fluorescence Spectra of 1 compared with the magnetic blocking barrier and ab initio calculation results. Note the higher energy transitions (grey arrows) involve the excited states of the ${ }^{4} \mathrm{~F}_{9 / 2}$ state. 
We also performed the photoluminescence measurement under a pulsed high magnetic field up to $37 \mathrm{~T}$ to probe its magnetic response. ${ }^{[12]}$ Although several weak peaks cannot be observed, the pulse-field emission still matches well with the conventional spectra. Significant effect of the Zeeman splitting is observed with the general shift towards lower wavenumber (owing to the ground-doublet splitting of the ${ }^{4} \mathrm{~F}_{9 / 2}$ state) and the further splitting into pairs of peaks within the ${ }^{6} \mathrm{H}_{15 / 2}$ state. We notice that the asymmetric character around $20900 \mathrm{~cm}^{-1}$ might indicate the overlapping of multiple peaks with different center positions and Zeeman splitting, which is agree with the close lying of the first and second excited state (No.2-3). Also, the small splitting of the strongest peak at $20914 \mathrm{~cm}^{-1}$ (No. 3) rules out its possibility to involve the ground doublet which is with the largest $g_{z}$ (see calculation for details). Here, the energy gaps from the ground doublet to these two excited doublets are 322 $\mathrm{cm}^{-1}$ and $396 \mathrm{~cm}^{-1}$, respectively, matching well with the spinreversal barrier of $353 \mathrm{~cm}^{-1}$ for 1 and $324 \mathrm{~cm}^{-1}$ for $1 @ \mathrm{Y}$ determined by the magnetic measurements.

\section{$A b$ Initio Calculation}

Further insight into the electronic structure and magnetic properties of 1 was obtained by quantum chemistry calculations. The geometry of $\mathbf{1}$ was extracted from the crystal structure and the positions of hydrogen atoms were optimized at DFT level. This was done because the coordinates of the hydrogen atoms refined from the diffraction data are usually not very accurate and the hydrogens of the coordinated water molecules lie close to the Dy ion and may therefore have an appreciable effect on the crystal field. The optimization retains the nearly planar pseudo- $D_{5 \mathrm{~h}}$ arrangement of the five water molecules. The structure used in the DFT and $a b$ initio calculations included, in addition to the complex $\mathbf{1}$, the outer coordination sphere containing the three bromide ions and the two free ligands hydrogen bonded into the coordinated water molecules (Fig. 1). Calculations were also conducted on various truncated structures but the results show that any simplification of the structure leads to qualitative changes in the energy spectrum and the magnetic properties.

Table 1. Calculated $\boldsymbol{g}$-tensors of the eight lowest Kramers doublets arising from the crystal field splitting of the ${ }^{6} \mathrm{H}_{15 / 2}$ manifold

\begin{tabular}{cccc}
\hline KD & $g_{\boldsymbol{x}}$ & $\boldsymbol{g}_{\boldsymbol{y}}$ & $\boldsymbol{g}_{\mathbf{z}}$ \\
\hline 1 & 0.0002 & 0.0002 & 19.8812 \\
2 & 0.9571 & 3.4268 & 16.6964 \\
3 & 0.0881 & 0.1268 & 16.9229 \\
4 & 3.3031 & 4.5458 & 8.7070 \\
5 & 1.4008 & 3.7771 & 6.5247 \\
6 & 0.2470 & 0.3874 & 13.6266 \\
7 & 0.2963 & 1.2140 & 11.2279 \\
8 & 0.5039 & 1.0113 & 13.1702 \\
\hline
\end{tabular}

The calculated $\boldsymbol{g}$-tensors of the eight lowest Kramers doublet are listed in Table 1 . The ground doublet is highly axial $\left(g_{x}=\right.$ $\left.0.0002, g_{y}=0.0002, g_{z}=19.8812\right)$ explaining the prominent SMM behavior of $\mathbf{1}$ at low temperatures. The orientations of the main anisotropy axes $\left(g_{z}\right)$ of the three lowest Kramers doublets are illustrated in Fig. 1a. The axis of the ground doublet is aligned roughly along the main rotational axis of the pseudo- $D_{5 \mathrm{~h}}$ symmetry around the Dy ion and deviates from the two axial Dy$\mathrm{O}$ bond vectors by an angle of $2.9^{\circ}$. The main axis of the first excited Kramers doublet lies along the $\mathrm{C}_{2}$ axis of the crystal structure and is near perfectly perpendicular to the ground axis with an angle of $90.0^{\circ}$ between them. The third axis lies again almost parallel to the main rotational axis with an angle of $0.3^{\circ}$ between it and the ground axis.

To elucidate the effects of the electronic structure on the relaxation of magnetization in $\mathbf{1}$ the magnetization blocking barrier was also studied using previously proposed methodology. ${ }^{[13 a]}$ The results are summarized in Fig. 4b and show that the most probable relaxation pathway takes place via the first excited Kramers doublet. The local pseudo- $D_{5 \mathrm{~h}}$ symmetry around the Dy ion leads to suppression of the QTM process indicated by the relatively small value of the matrix element connecting the two components of the ground doublet. However, the lower symmetry introduced by the outer coordination sphere leads to rotation of the main magnetic axis of the first excited doublet into an orientation parallel to the true $\mathrm{C}_{2}$ axis of the crystal structure which lies perpendicular to the ground axis. As previously demonstrated, the large angle between the two Kramers doublets leads to large transverse magnetic matrix elements between the components of the respective doublets. ${ }^{[13 b]}$ This allows the magnetization to traverse through the barrier and leads to lowering of the blocking barrier as compared to an analogous complex in ideal $D_{5 \mathrm{~h}}$ symmetry.

Although the properties of Kramers doublets - such as the $g$ tensors and transition magnetic moments - are well reproduced at CASSCF level, quantitative comparison of the calculated energy levels with those obtained from the luminescence spectrum require explicit treatment of dynamic correlation. This can be achieved by applying a second order correction to the CASSCF energies using the extended multistate (XMS) CASPT2 method. ${ }^{[13 c]}$ Treatment of the full molecular structure, including the outer coordination sphere, at this level is not feasible in terms of computational costs and simplification of the structure may result into qualitative differences in the calculated energetics. Thus, dynamic correlation effects were treated as a correction to the ab initio crystal field potential of the ${ }^{6} \mathrm{H}_{15 / 2}$ ground multiplet (see the supporting information for detailed description of the procedure) and a corrected crystal field Hamiltonian was diagonalized to obtain the final spectrum. The crystal field corrected energies of the eight lowest Kramers doublets are presented in Table S4 along with the respective values calculated at CASSCF level for the full geometry and at CASSCF and XMS-CASPT2 levels for a truncated geometry where the phenyl and cyclohexyl groups of the ligands have been replaced with methyl groups. The results clearly show that dynamic correlation and the full structure are required to approach the experimental levels.

The energy of the first excited Kramers doublet calculated using the crystal field correction approach is $297 \mathrm{~cm}^{-1}$ which is in good agreement with the energy difference of $322 \mathrm{~cm}^{-1}$ obtained 
from the luminescence spectrum. The value is also in semiquantitative agreement with the experimental barrier heights 353 $\mathrm{cm}^{-1}$ for 1 and $324 \mathrm{~cm}^{-1}$ for $1 @ Y$ from magnetism. Agreement with the higher excited states is poorer. This deviation most likely results from the approximate nature of the crystal field correction procedure employed in this study. We expect that enlarging the active space of the CASSCF method by explicitly correlating several ligand type orbitals and/or metal's occupied and virtual orbitals would increase the quality of the reference wave function, leading to an even closer agreement for the excited states. This was recently demonstrated for Er-trensal complex, ${ }^{[14]}$ for which a larger active space increased the overall agreement for each of the crystal field levels of the ground $J=$ $15 / 2$ multiplet, as well as for their properties.

\section{Conclusions}

In conclusion, while preserving the large energy barrier and high hysteresis temperature, we successfully performed the singlecrystal micro-SQUID measurement at ultra-low temperature and introduced the characteristic lanthanide photoluminescence into the family of pentagonal bipyramidal Dy(III) single-ion magnets based on phosphine oxide. The AC, DC and micro-SQUID relaxation data follow in the same trend and the hysteresis loops become wider when going down to $0.04 \mathrm{~K}$. The fluorescence spectra provide crucial information of the energy levels that correspond well with the magnetic measurements and shows semi-quantitative agreement with the $a b$ initio calculation. It should be pointed out that the magnetic blocking in many of these SMMs with high energy barriers is controlled by the Raman process rather than the Orbach process, which requires deeper understanding in the future. Apart from this, we also note that the fast QTM around zero field sets up a limit for the further increase of the relaxation time. Efforts regarding this could be focus on the fine-tuning of the magnetic interactions and explore the lanthanide nuclides of suitable nuclear spins into an SIM/SMM system.

\section{Experimental Section}

General Procedure All reagents were commercially available and used as received without further purification. The elemental analyses were performed with an Elementar Vario-EL CHN elemental analyzer. The IR spectra were recorded using a Thermo Nicolet AVATAR 330 FT-IR spectrometer. The powder XRD patterns were recorded on a Bruker D8 $\mathrm{X}$-Ray diffractometer with $\mathrm{Cu} \mathrm{Ka}$ radiation. Thermogravimetric analysis (TGA) was carried out on a NETZSCH TG209F3 thermogravimetric analyzer. The ICP-AES analyses were performed with a TJA IRIS (HR) spectrometer.

Synthesis. [Dy $\left.\left(\mathrm{CyPh}_{2} \mathrm{PO}\right)_{2}\left(\mathrm{H}_{2} \mathrm{O}\right)_{5}\right] \mathrm{Br}_{3} \cdot 2\left(\mathrm{CyPh}_{2} \mathrm{PO}\right) \cdot \mathrm{EtOH} \cdot 3 \mathrm{H}_{2} \mathrm{O}$ (1) are synthesized by dissolving Hydrate $\mathrm{DyBr}_{3}(0.2 \mathrm{mmol})$ and $\mathrm{CyPh}_{2} \mathrm{PO}(0.4$ $\mathrm{mmol}$ ) in a $\mathrm{H}_{2} \mathrm{O} / \mathrm{EtOH}(1: 9,4 \mathrm{~mL})$ mixed solution, followed by the slow evaporation in ambient condition to almost dryness. Then $2 \mathrm{~mL} E \mathrm{EtOH}$ was added for recrystallization. Colorless crystals suitable for X-ray analysis began to grow after 3 days, which are then collected by filtration, washed with cold $\mathrm{EtOH}$ and then dried in air (yield ca. 40\%). The disordered solvent molecules in the voids were unable to be determined by single-crystal diffraction but confirmed via elemental analysis and thermogravimetric analysis. Analysis (calcd., found) for $\mathrm{C}_{76} \mathrm{H}_{112} \mathrm{P}_{4} \mathrm{Br}_{3} \mathrm{DyO}_{14}$ (1): C (51.40, 51.47), H (6.35, 6.02), IR( $\left(\mathrm{cm}^{-1}\right)$ : 3275br, 2933s, 2852s, 1632m, 1485m, 1439s, 1176s, 1135s, 1120s, 1095s, $999 \mathrm{~m}, 887 \mathrm{~m}, 746 \mathrm{~s}, 725 \mathrm{~s}, 700 \mathrm{~s}, 557 \mathrm{~s}, 536 \mathrm{~s}$. The diluted samples 1@Y was synthesized in the same way with $\mathrm{DyBr}_{3}: \mathrm{YBr}_{3}=1: 19$. The dilution ratios were confirmed by both the magnetization (ca. $4 \%$ ) and ICP-AES analyses $(5 \% \pm 0.5 \%)$, and the phase purity was checked by the powder XRD. Analysis (calcd., found) for $\mathrm{C}_{76} \mathrm{H}_{112} \mathrm{P}_{4} \mathrm{Br}_{3} \mathrm{Y}_{0.95} \mathrm{Dy}_{0.05} \mathrm{O}_{14}$ (1@Y): C (53.51, 53.61), H (6.62, 6.21), IR $\left(\mathrm{cm}^{-1}\right): 3256 \mathrm{br}, 2933 \mathrm{~s}, 2852 \mathrm{~s}, 1633 \mathrm{~m}$, $1487 \mathrm{~m}, 1439 \mathrm{~s}, 1144 \mathrm{~s}, 1121 \mathrm{~s}, 1092 \mathrm{~s}, 999 \mathrm{~m}, 889 \mathrm{~m}, 825 \mathrm{~m}, 747 \mathrm{~s}, 727 \mathrm{~s}$, $698 \mathrm{~s}, 557 \mathrm{~s}, 534 \mathrm{~s}$

X-ray Crystallography Single-crystal diffraction data was recorded at 150(2) K on a Rigaku R-AXIS SPIDER Image Plate diffractometer with Mo Ka radiation, solved by direct methods and refined using SHELXTL program. ${ }^{[15]}$ Crystal Data and Structural Refinement are listed in Supplementary Table S1-S3. CCDC 1519258-1519259 contain the supplementary crystallographic data for this paper. These data can be obtained free of charge from the Cambridge Crystallographic Data Centre via www.ccdc.cam.ac.uk/data_request/cif.

Magnetic Measurements The dc and ac magnetic measurements were performed on the polycrystalline samples using a Quantum Design MPMS XL-7 SQUID magnetometer. The ZFC-FC $(0.1 \mathrm{~T}, 2 \mathrm{~K} / \mathrm{min})$, hysteresis $(0.02 \mathrm{~T} / \mathrm{s})$ and relaxation were measured on a Quantum Design PPMS with VSM option. The single-crystal measurements down to ultra-low temperature were performed on a micro-SQUID. ${ }^{16]}$ Diamagnetic correction was performed based on the Pascal's coefficients In the lower temperature region, the relaxation rate is determined by measuring the dc magnetization decay after the removal of a saturated field of $3 \mathrm{~T}$.

Optical Measurements The luminescence spectra were recorded on an Edinburgh FLS-980 Fluorescence spectrometer equipped with Xenon light, PMT detector and ALS cryostat down to $10 \mathrm{~K}$. The slit and step were set to $0.1 \mathrm{~nm}$ to capture the detailed splitting of the peaks. The pulsed high magnetic field photoluminescence measurement was performed at Wuhan National High Magnetic Center with a laser-fiber spectrometer setup operating at $5 \mathrm{~K}$. The magnitude of magnetic field is detected at the falling side of a magnetic pulse and each luminescence spectrum was captured within $1 \mathrm{~ms}$.

\section{Acknowledgements}

This work was supported by the "973 Project" (2014CB845602), the NSFC (Grant nos 21620102002, 21371183 and 91422302), and the NSF of Guangdong (S2013020013002).

Keywords: lanthanide $\cdot$ dysprosium $\bullet$ magnetic properties $•$ single-molecule magnets $•$ Fluorescence

[1] a) D. Gatteschi, R. Sessoli, J. Villain, Molecular nanomagnets, Oxford University Press, 2006; b) M. N. Leuenberger, D. Loss, Nature 2001, 410, 789-793; c) W. Wernsdorfer, Science 1999, 284, 133-135; d) L. Bogani, W. Wernsdorfer, Nat. Mater. 2008, 7, 179-186; e) M. Mannini, F. Pineider, P. Sainctavit, C. Danieli, E. Otero, C. Sciancalepore, A. M. Talarico, M.-A. Arrio, A. Cornia, D. Gatteschi, R. Sessoli, Nat. Mater. 2009, 8, 194-197; f) M. Ganzhorn, S. Klyatskaya, M. Ruben, W. Wernsdorfer, Nat. Nano. 2013, 8, 165-169. 
[2] R. Sessoli, D. Gatteschi, A. Caneschi, M. A. Novak, Nature 1993, 365 , 141-143.

[3] a) S. Osa, T. Kido, N. Matsumoto, N. Re, A. Pochaba, J. Mrozinski, J. Am. Chem. Soc. 2003, 126, 420-421; b) N. Ishikawa, M. Sugita, T. Ishikawa, S.-y. Koshihara, Y. Kaizu, J. Am. Chem. Soc. 2003, 125, 8694-8695; c) D. N. Woodruff, R. E. P. Winpenny, R. A. Layfield, Chem Rev. 2013, 113, 5110-5148; d) R. J. Blagg, L. Ungur, F. Tuna, J. Speak, P. Comar, D. Collison, W. Wernsdorfer, E. J. L. Mclnnes, L. F Chibotaru, R. E. P. Winpenny, Nat. Chem. 2013, 5, 673-678; e) S.-D. Jiang, B.-W. Wang, H.-L. Sun, Z.-M. Wang, S. Gao, J. Am. Chem. Soc 2011, 133, 4730-4733; f) S. K. Langley, D. P. Wielechowski, V. Vieru, N. F. Chilton, B. Moubaraki, B. F. Abrahams, L. F. Chibotaru, K. S. Murray, Angew. Chem. Int. Ed. 2013, 52, 12014-12019.

[4] a) J. D. Rinehart, M. Fang, W. J. Evans, J. R. Long, Nat. Chem. 2011, 3, 538-542; b) J. D. Rinehart, M. Fang, W. J. Evans, J. R. Long, J. Am. Chem. Soc. 2011, 133, 14236-14239; c) S. Demir, J. M. Zadrozny, M. Nippe, J. R. Long, J. Am. Chem. Soc. 2012, 134, 18546-18549.

[5] a) J. M. Zadrozny, D. J. Xiao, M. Atanasov, G. J. Long, F. Grandjean, F. Neese, J. R. Long, Nat. Chem. 2013, 5, 577-581; b)J. M. Zadrozny, J. R. Long, J. Am. Chem. Soc. 2011, 133, 20732-20734; c) R. C. Poulten, M. J. Page, A. G. Algarra, J. J. Le Roy, I. López, E. Carter, A. Llobet, S. A. Macgregor, M. F. Mahon, D. M. Murphy, M. Murugesu, M. K. Whittlesey, J. Am. Chem. Soc. 2013, 135, 13640-13643; d) R Westerström, J. Dreiser, C. Piamonteze, M. Muntwiler, S. Weyeneth, H. Brune, S. Rusponi, F. Nolting, A. Popov, S. Yang, L. Dunsch, T. Greber, J. Am. Chem. Soc. 2012, 134, 9840-9843; e) S.-D. Jiang, B.-W. Wang, G. Su, Z.-M. Wang, S. Gao, Angew. Chem. Int. Ed. 2010, 49, 7448 7451 ; f) N. F. Chilton, S. K. Langley, B. Moubaraki, A. Soncini, S. R. Batten, K. S. Murray, Chem. Sci. 2013, 4, 1719-1730.

[6] C. Görller-Walrand, K. Binnemans, in Handbook on the Physics and Chemistry of Rare Earths, Vol. Volume 23 (Eds.: Karl A. Gschneidner, Jr., E. LeRoy), Elsevier, 1996, pp. 121-283.

[7] M. A. AIDamen, J. M. Clemente-Juan, E. Coronado, C. Martí-Gastaldo, A. Gaita-Ariño, J. Am. Chem. Soc. 2008, 130, 8874-8875.
[8] a) K. R. Meihaus, J. R. Long, J. Am. Chem. Soc. 2013, 135, 17952 17957. b) L. Ungur, J. J. Le Roy, I. Korobkov, M. Murugesu, L. F. Chibotaru, Angew. Chem. Int. Ed. 2014, 53, 4413-4417.

[9] a) J.-L. Liu, Y.-C. Chen, Y.-Z. Zheng, W.-Q. Lin, L. Ungur, W. Wernsdorfer, L. F. Chibotaru, M.-L. Tong, Chem. Sci. 2013, 4, 33103316; b) J.-L. Liu, J.-Y. Wu, Y.-C. Chen, V. Mereacre, A. K. Powell, L. Ungur, L. F. Chibotaru, X.-M. Chen, M.-L. Tong, Angew. Chem. Int. Ed. 2014, 53, 12966-12970; c) Y.-C. Chen, J.-L. Liu, L. Ungur, J. Liu, Q.-W Li, L.-F. Wang, Z.-P. Ni, L. F. Chibotaru, X.-M. Chen, M.-L. Tong, J. Am. Chem. Soc. 2016, 138, 2829-2837; d) J. Liu, Y.-C. Chen, J.-L. Liu, V. Vieru, L. Ungur, J.-H. Jia, L. F. Chibotaru, Y. Lan, W. Wernsdorfer, S. Gao, X.-M. Chen, M.-L. Tong, J. Am. Chem. Soc. 2016, 138, 5441 5450 .

[10] a) S. Cardona-Serra, J. M. Clemente-Juan, E. Coronado, A. GaitaAriño, A. Camón, M. Evangelisti, F. Luis, M. J. Martínez-Pérez, J. Sesé, J. Am. Chem. Soc. 2012, 134, 14982-14990; b) S. K. Gupta, T. Rajeshkumar, G. Rajaraman, R. Murugavel, Chem. Sci. 2016, 7, 51815191; c) Y.-S. Ding, N. F. Chilton, R. E. P. Winpenny, Y.-Z. Zheng, Angew. Chem. Int. Ed. 2016, DOI: 10.1002/anie.201609685.

[11] M. Gregson, N. F. Chilton, A.-M. Ariciu, F. Tuna, I. F. Crowe, W. Lewis, A. J. Blake, D. Collison, E. J. L. Mclnnes, R. E. P. Winpenny, S. T. Liddle, Chem. Sci. 2016, 7, 155-165.

[12] Y. Bi, C. Chen, Y.-F. Zhao, Y.-Q. Zhang, S.-D. Jiang, B.-W. Wang, J.-B. Han, J.-L. Sun, Z.-Q. Bian, Z.-M. Wang, S. Gao, Chem. Sci. 2016, 7 5020-5031.

[13] a) L. Ungur, M. Thewissen, J.-P. Costes, W. Wernsdorfer, L. F. Chibotaru, Inorg. Chem. 2013, 52, 6328-6337; b) L. Ungur, L. F. Chibotaru, Phys. Chem. Chem. Phys. 2011, 13, 20086-20090; c) T. Shiozaki, W. Győrffy, P. Celani, H.-J. Werner, J. Chem. Phys. 2011, 135, 081106 .

[14] L. Ungur, L. F. Chibotaru, Chem. Eur. J., 2016. DOI: 10.1002/chem.201605102

[15] G. Sheldrick, Acta Crystallographica Section A 2008, 64, 112-122.

[16] W. Wernsdorfer, Supercond. Sci. Technol. 2009, 22, 064013. 


\section{Entry for the Table of Contents}

\section{FULL PAPER}

Dynamic magnetism and magnetooptical correlation are firstly studied for a pentagonal bipyramidal Dy(III) single-ion magnet based on fluorescence-active phosphine oxide with large $U_{\text {eff }}=508(2) \mathrm{K}$ and high magnetic hysteresis temperature of 19 $\mathrm{K}$.

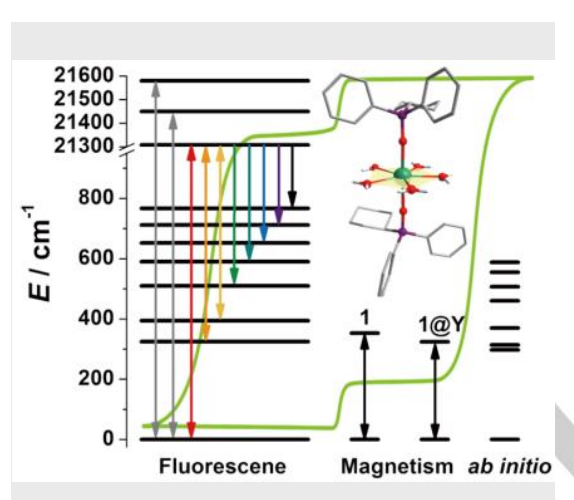

Yan-Cong Chen, Jun-Liang Liu, *

Yanhua Lan, Zhi-Qiang Zhong, Akseli Mansikkamäki, Liviu Ungur, Quan-Wen Li, Jian-Hua Jia, Liviu F. Chibotaru, JunBo Han, Wolfgang Wernsdorfer, XiaoMing Chen, and Ming-Liang Tong*

Page No. - Page No.

Dynamic Magnetic and Optical Insight into a High Performance Pentagonal Bipyramidal Dy(III) Single-Ion Magnet 